\title{
Development of Eco-Friendly Soap-Based Firefighting Foam for Forest Fire
}

\author{
Takayoshi KawAHARA ${ }^{1-3}$, Shuichi HATAE ${ }^{1}$, Takahide KANYAMA ${ }^{1,2}$, Yuki IshizAKI ${ }^{4}$ and Kazuya UezU ${ }^{2,4}$ \\ ${ }^{\prime}$ Research and Development Department, Shabondama Soap Co., Ltd., Kitakyushu, Fukuoka 808-0195, Japan \\ ${ }^{2}$ Soap Research Center, Kitakyushu, Fukuoka 808-0138, Japan \\ ${ }^{3}$ Department of Microbiology, University of Occupational and Environmental Health, Kitakyushu, Fukuoka 807-8556, Japan \\ ${ }^{4}$ Department of Chemistry and Biochemistry, University of Kitakyushu, Kitakyushu, Fukuoka 808-0135, Japan
}

(Received September 1, 2015; Accepted November 9, 2015)

\begin{abstract}
Recently, large-scale forest fires have been occurring frequently worldwide. In forest fires, the use of the firefighting foam has greatly improved fire extinguishing effects, but the environmental impact from firefighting activities using foam still cause concerns. We have been developing a novel firefighting foam for forest fire with significantly lower environmental risk, consisting of soaps, chelating agent, and diluents. We determined the optimal composition of soap, and found the high biodegradable chelating agent with a high foaming performance.
\end{abstract}

Keywords : environmental-friendly, extinguishing agent, fatty acid salts, wildfire

\section{INTRODUCTION}

In recent years, large-scale forest fires have been occurring frequently worldwide and 60,000 to $140,000 \mathrm{~km}^{2}$ of forests have been vanishing yearly. The forest fires and the damage from the smoke associated with them have caused lost of human life and health hazards as well as loss of forest vegetation, biomass, and ecosystems in massive areas. The damage in the Europe's Mediterranean coast, North America's west coast, Russia, and Australia are especially serious. There were over 170 large-scale forest fires concentrated throughout Greece from June to October in 2007. Additionally, in 2007 the forest fires that broke out from October 20 to 23 in Southern California of North America over an area almost equivalent to the size of Tokyo $(2,187$ $\mathrm{km}^{2}$ ) destroyed approximately $2,000 \mathrm{~km}^{2}$ of forests and about 1,700 homes in $7 \mathrm{~d}$ time until October 27 (Hayasaka, 2010).

In these forest fires, the use of firefighting foam has greatly improved fire extinguishing effects. The surfactant in firefighting foam significantly lowers surface tension in water and allows water to be efficiently adherent to flammable materials and additionally allows water to penetrate into the flammable materials. Because of this, it is highly effective in firefighting efforts by the increased cooling effect, suppression of oxygen supply, suppression of chemical reactions in the flammable material, and blockage of radiated heat, etc. However, for forest fires, the use of water alone is common in extinguishing activities. This is because the mountainous areas are also the source of water for urban residents and the pollution caused by the run-off from the firefighting foam into rivers and the surrounding environment would be especially concerning. A cautious stance in the use of firefighting foam is being taken and even in the United States, therefore, there was a case where the concern regarding secondary environmental damage from firefighting foam led to allowing fires to extinguish naturally and resulted in a large area of new and old forests to be destroyed.

We have been developing firefighting foam involving naturally derived fatty acid salts (soap) as the main component. The soap is much lower in toxicity to aquatic organisms compared to synthetic detergents with environmental water in river, lake and sea (Lin et al., 2006; Kawano et al., 2007; Goto et al., 2007; 2008; Kawano et al., 2014). The reason why the toxicity of the soap is lower to aquatic microorganisms and fish is that it reacts with metal ions such as calcium ions that exist in the environment to form metal soap. The metal soap does not have the interfacial activity and its toxicity is significantly low.

It is desirable to use soap that has very low toxicity to aquatic organisms since the spreading fire extinguishing agents could have a large impact on the natural environment. The soap-based firefighting foam is diluted by 1 wt $\%$ with environmental water in use. The metal ions such as calcium ions in the environmental water may cause the forming of metal soap, and the interfacial activity may be lowered. Therefore, it is necessary to add a chelating agent in the firefighting foam to prevent soap and metal ions from forming metal soap. Ethylenediaminetetraacetic acid (EDTA), commonly used chelating agent, is very effective in masking metal ions. However, EDTA is much less biodegradable and its long-term residual in the environment, particularly as heavy metal complex, raises concerns about ecotoxicities (Oviedo et al., 2003). For this reason, the highly biodegradable chelating agent, L-glutamic acid N,Ndiacetic acid (GLDA), was used in firefighting foam for general buildings (Mizuki et al., 2007). However, the firefighting foam with GLDA suffered from the disadvan-

Corresponding author: Kazuya Uezu, fax: +81-93-695-3368, e-mail : uezu@kitakyu-u.ac.jp 
tage of high kinetic viscosity and low fluidity at low temperature.

In this study, we focused on the counter cations of fatty acid salts and chelating agents to develop the soapbased firefighting foam with lower kinetic viscosity. The chelating agents were selected among the highly biodegradable chelating agents (Pinto et al., 2014) from the perspective of the impact on the environment.

\section{MATERIALS AND METHODS}

\section{Composition of soap-based firefighting foam}

Firefighting foam is composed of a soap component, chelating agent and diluent; in this work we have been used a proportion of respectively 17.16, 16 and 66.84 (wt\%). Potassium laurate (LauK), potassium oleate (OleK), and sodium oleate $(\mathrm{OleNa})$ were used as the soap component. Lauric acid and oleic acid was purchased from Cognis Oleochemicals Co., Ltd., Malaysia and Miyoshi Oil \& Fat Co., Ltd., Japan to prepare lauK, OleK and OleNa. Methyl glycine diacetic acid (MGDA, manufactured by BASF, Germany), L- glutamic acid diacetic acid (GLDA, manufactured by Chelest Corporation, Japan), Ethylenedinitrilotetraacetic acid (EDTA, manufactured by Chelest Corporation, Japan), Nitrilotriacetic acid (NTA, manufactured by Chelest Corporation, Japan), sodium succinate (DS, manufactured by LANXESS Co., Ltd., Germany) and iminodisuccinic acid (IDS, manufactured by LANXESS Corporation, Germany) were used for chelating agents. Water, propylene glycol (PG) and xylene glycol (HG) were used as diluents.

Pour point

This test was based on Japanese Industrial Standards K2269 (the pour point test method). Ice, sodium chloride, and dry ice were used to lower the cooling bath temperature. $45 \mathrm{~mL}$ of the sample was poured up to the test tube's maker height and was sealed. The test tube was installed in the outer tube and warmed up to $45^{\circ} \mathrm{C}$. The sample was retained for $5 \mathrm{~s}$ at the preset temperature. The pour point is defined as the temperature at which the sample lost the fluidity (Mizuki et al., 2007).

Foaming test

$4 \mathrm{~L}$ of Class A foam solution at $20 \pm 2^{\circ} \mathrm{C}$ was sealed into the fire pump. Next, nitrogen gas was poured in the fire pump up to $0.85 \mathrm{MPa}$. The solution was drained off from the fire pump to a bubble collection tank $(\phi 200 \mathrm{~mm}$ $\times h 1.2 \mathrm{~m})$. The steady-state foam height $(h, \mathrm{~cm})$ was measured as the distance between the bottom of the tank and the top of the foam. The Expansion ratio was calculated according to Eq. 1 (Mizuki et al., 2007).

$$
\begin{aligned}
& \text { Expansion ratio }= \\
& \frac{(\text { foam height })(\text { tank cross }- \text { section area) }}{\text { (volume of the aqueous solution) }}
\end{aligned}
$$

\section{Kinetic viscosity}

Measurements were carried out using the Kinematic Viscosity Test Method and Viscosity Index Calculation Method as dictated in JIS K 2283 for crude oil petroleum and petroleum products. The time $(t)$ for the upper edge of the sample flows from the measurement time evaluation line (mark) $\mathrm{E}$ to $\mathrm{F}$ of the Cannon-Fenske Kinematic Viscometer was measured and kinetic viscosity (v) was calculated according to Eq.2.

$$
v=\mathrm{C}(\text { kinematic viscosity constant }) \times t(\mathrm{~s})
$$

\section{RESULTS AND DISCUSSION}

\section{Selection of chelating agents}

The expansion ratios of the soap-based firefighting foam with MGDA, GLDA, EDTA, NTA, DS and IDS as chelating agent were shown in Fig 1. When lauric acid and oleic acid proportions were $4: 6$ by weight, the fluidity and the expansion ratio were very high (Mizuki et al., 2007). Therefore, we determined that the proportion of LauK: OleK is $4: 6$ in this research.

Very high expansion ratio was observed for the soapbased firefighting foam with EDTA and MGDA (9.7 times for EDTA and 9.6 times for MGDA); high expansion ratio for the firefighting foam with NTA and GLDA (6.3 times for NTA and 5.6 times for GLDA); low expansion ratio for the firefighting foam with DS and IDS (2.6 times for DS and 2.3 times for IDS). Since the expansion ratio is required more than 5 times according to Japan's foam extinguisher test standards, the firefighting foams with EDTA, MGDA, NTA, and GLDA were found to satisfy the standards. However, EDTA has poor biodegradability and the risk of long-term residual in the environment to cause ecotoxicity, and NTA is highly toxic (Pinto et al., 2014). Forest fire extinguishing agents are sprayed in large quantities toward the natural environment, and their influence on the environment should be carefully considered. Therefore, the chelating agents used in firefighting foams for forest fire should be highly biodegradable and very low in toxicity to living organisms. MGDA is known to be a chelating agent with good biodegradability and expected to have little impact on the environment (Pinto et al., 2014). For this reason, it was decided that MGDA would be used as a chelating agent for the soap-based firefighting foams for forest fires.

Selection of counter cations

When the proportion of lauric acid salt and oleic acid

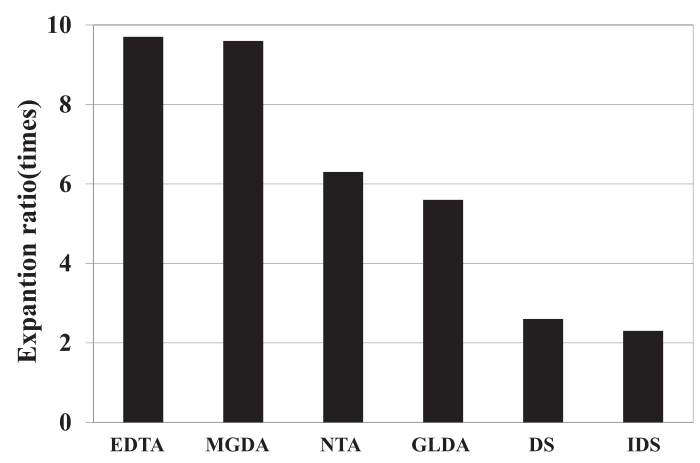

Fig. 1 The expansion ratio of firefighting foams including different chelating agents. Potassium ion was used as a counter ion. Fatty acid salt composition was potassium laurate $(\mathrm{LauK})$ : potassium oleate $(\mathrm{OleK})=4: 6$. 
salt is $4: 6$ by weight, the pour point is the lowest for the soap-based firefighting foam for general buildings (Mizuki et al., 2007). According to the result, the pour point was measured for the weight ratios LauK:OleK $=4: 6$ and $\mathrm{LauK}: \mathrm{OleNa}=4: 6$. It was found that the pour point of the firefighting foam with potassium ion as counter cation of oleic acid was lower by $5^{\circ} \mathrm{C}$ or more with sodium ion as counter cation (Fig. 2). In general, it is thought that since the solubility of potassium ions is high, the pour point is lowered.

Additionally, it is known that the mixture of sodium oleate and GLDA is easy to gelate since a giant lamellar structure is formed by the sodium ion cross-linking (Jeong et al., 2007). It can be considered to suppress gelling and lower the pour point by replacing the chelating agent and the counter ion from GLDA and sodium ion to MGDA and potassium ion due to different molecular and ion size.

Optimization of fatty acid composition

MGDA was used as the chelating agent, LauK and OleK were used as the soap component. The mix ratio of LauK and OleK was changed and the results of measuring the expansion ratio are shown in Fig. 3. When the proportion of OleK is increased, the expansion ratio becomes higher, and at around $60 \%$ it showed the maximum (9.6 times). This is different from the results of Mizuki et al. (2007) where the expansion ratio lowers when sodium oleate is increased. This is because of the formation of gelling of OleNa and GLDA (Jeong et al., 2007) and when the proportion of oleic acid is increased, the viscosity of the liquid increases. It can be considered that when the viscosity of the liquid increases, the movement of the soap component toward the air-water interface is suppressed, and consequently the expansion ratio is lowered. Since the vis-

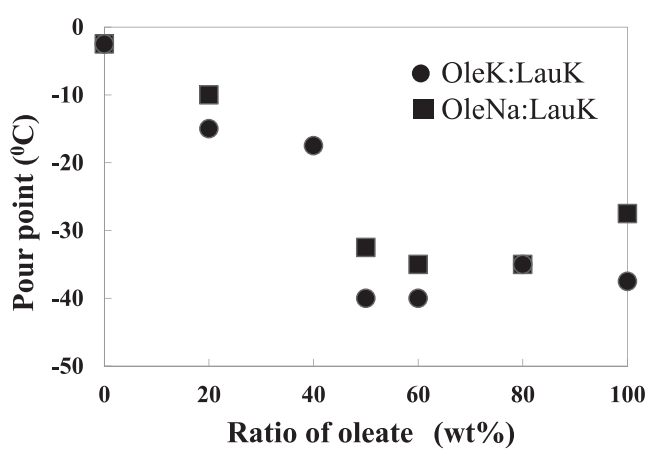

Fig. 2 The pour point of firefighting foams with various ratios of potassium laurate (LauK) and potassium oleate (OleK) or sodium oleate (OleNa). MGDA was used for the chelating agent. cosity of the firefighting foam was decreased by changing the chelating agent and the counter ion to MGDA and potassium, the influence of viscosity of the liquid is small and resulted in the different behavior from the results of Mizuki et al. From these results, we confirmed that the fatty acid salt composition $\mathrm{LauK}: \mathrm{OleK}=4: 6$ is appropriate for the soap-based firefighting foam.

Comparison of firefighting foam for structural fire and forest fire

In order to solve the problem of viscosity of firefighting foams for forest fires, low viscosity and high expansion ratio were achieved by changing the chelating agent and the counter ion. The physical properties of the conventional product and the forest fire extinguishing agent developed in this study are summarized in Table 1.

We succeeded in lowering the pour point by $7.5^{\circ} \mathrm{C}$ which is the index for low temperature stability, and in lowering the kinematic viscosity by $8 \mathrm{cSt}$ at the assumed temperature for normal use of $30^{\circ} \mathrm{C}$ and by $110 \mathrm{cST}$ or more at the assumed temperature used in cold climates of $-10^{\circ} \mathrm{C}$. We considered that the firefighting foam for forest fires has better operability and is easier to use than general building firefighting foams.

\section{CONCLUSIONS}

In order to develop the eco-friendly soap-based firefighting foam for forest fires, we examined counter ions and cheating agents. By screening for highly biodegradable chelating agents, we found that the firefighting foam with MGDA showed better performance in expansion ratio, operability, and low temperature stability. By using potassium ion instead of sodium ion as the counter cation of fatty acid salt, the kinetic viscosity and the pour point were

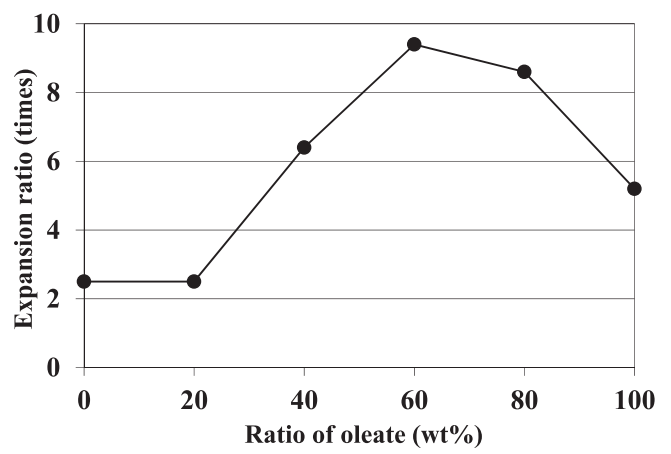

Fig. 3 The expansion ratio of firefighting foams with various ratios of potassium laurate (LauK) and potassium oleate (OleK). MGDA was used as a chelating agent.

Table 1 Comparison between the firefighting foam developed in this study for forest fire and the conventional firefighting foam for structural fire.

\begin{tabular}{|c|c|c|c|c|}
\hline & \multirow[b]{2}{*}{$\begin{array}{c}\text { Expansion ratio } \\
\text { Times }\end{array}$} & \multirow[b]{2}{*}{$\begin{array}{l}\text { Pour point } \\
{ }^{\circ} \mathrm{C}\end{array}$} & \multicolumn{2}{|c|}{ Kinetic viscosity } \\
\hline & & & $\begin{array}{c}\text { at }-10^{\circ} \mathrm{C} \\
\mathrm{cSt}\end{array}$ & $\begin{array}{c}\text { at } 30^{\circ} \mathrm{C} \\
\mathrm{cSt}\end{array}$ \\
\hline $\begin{array}{l}\text { firefighting foam } \\
\text { for forest fire }\end{array}$ & 9.6 & -40.0 & 274.5 & 23.6 \\
\hline $\begin{array}{l}\text { firefighting foam } \\
\text { for structural fire }\end{array}$ & 5.6 & -32.5 & 390.0 & 32.0 \\
\hline
\end{tabular}


lowered, and the operability and the low temperature stability were increased.

In other words, a Class A firefighting foam for forest fires should be composed of soap as a surfactant for high biodegradability and low aquatic toxicity, and MGDA as a highly biodegradable chelating agent to suppress formation of metal soap. We succeeded in developing an environmentally-friendly firefighting foam for forest fires that will have little effect on the natural environment.

\section{ACKNOWLEDGEMENTS}

This work was supported by the Science and Technology Incubation Program "Development of soap-based firefighting foam for a forest fire", Advanced Region, Japan Science and Technology Agency.

\section{REFERENCES}

Goto, K., Kadono, T., Kawano, T. 2008. Use of natural mineral waters as the sources of diversified natural waters worldwide for testing the eco-toxicity of detergents using Green paramecia. ITE-IBA Lett. Batter. New Technol. Medic. 1: 184-188.

Goto, K., Lin, C., Kadono, T., Hirono, M., Uezu, K., Kawano, T. 2007. Eco-toxicity of a soap component (sodium oleate) and a synthetic detergent cocktail using green paramecia assayed in East Asia. J. Environ. Eng. Manag. 17: 377-383.

Hayasaka, Y. 2010. 2008 Forest fire in the Northern California, USA. (in Japanese) Bull. Jpn. Assoc. Fire Sci. Eng. 60: 1-9.
Jeong, Y., Uezu, K., Kobayashi, M., Sakurai, S., Masunaga, H., Inoue, K., Sasaki, S., Shimada, N., Tkeda, Y., Kaneko, K., Sakurai, K. 2007. Complex made from tetrasodium N,Nbis (carboxylatomethyl) glutamate and sodium oleate that forms a highly ordered lamella in gel phase. Bull. Chem. Soc. Jpn. 80: 410-417.

Kawano, T., Lin, C., Kadono, T., Uezu, K. 2007. Ecological risk assessment of fire-fighting chemicals using medaka fish (Oryzias latipes) in different water conditions. ITE-IBA Lett. Batter. New Technol. Medic. 8: 306-311.

Kawano, T., Otsuka, K., Kadono, T., Inokuchi, R., Ishizaki, Y., Dewancker, B., Uezu, K. 2014. Eco-toxicological evaluation of fire-fighting foams in small-sized aquatic and semiaquatic biotopes. Adv. Materials Res. 875-877: 699-707.

Lin, C., Kadono, T., Yoshizuka, K., Uezu, K., Kawano, T. 2006. Assessing the eco-toxicity of novel soap-based fire-fighting foam using medaka fish (Oryzias latipes, Red-orange variety) adopted to river and sea water conditions. ITE-IBA Lett. Batter. New Technol. Medic. 7: 499-503.

Mizuki, H., Uezu, K., Kawano, T., Kadono, T., Kobayashi, M., Hatae, S., Oba, Y., Iwamoto, S., Mitumune, S., Nagatomo, Y., Owari, Y., Umeki, H., Ymagaga, Y. 2007. Novel environmental friendly soap-based fire-fighting agent. J. Environ. Eng. Manage. 16: 403-408.

Oviedo, C., Rodriguez, J. 2003. EDTA: the chelating agent under environmental scrutiny. Quim. Nova 26: 901-905.

Pinto, I. S. S., Neto, I. F. F., Soares, H. M. V. Y. 2014. Biodegradable chelating agents for industrial, domestic, and agricultural applications - a review. Environ. Sci. Pollut. Res. 21: $11893-11906$ 